\title{
Stability of liquid bridges between equal disks in an axial gravity field
}

\author{
Lev A. Slobozhanin \\ Institute for Low Temperature Physics and Engineering, Ukrainian Academy of Sciences, 47 Lenin Ave., \\ 310164 Kharkov, Ukraine \\ Jose $M$. Perales \\ Lamf/ETSIA, Laboratorio de Aerodinámica. E.T.S.I. Aeronáuticos, Universidad Politécnica, \\ 28040 Madrid, Spain
}

\begin{abstract}
The stability of axisymmetric liquid bridges spanning two equal-diameter solid disks subjected to an axial gravity field of arbitrary intensity is analyzed for all possible liquid volumes. The boundary of the stability region for axisymmetric shapes (considering both axisymmetric and nonaxisymmetric perturbations) have been calculated. It is found that, for sufficiently small Bond numbers, three different unstable modes can appear. If the volume of liquid is decreased from that of an initially stable axisymmetric configuration the bridge either develops an axisymmetric instability (breaking in two drops as already known) or detaches its interface from the disk edges (if the length is smaller than a critical value depending on contact angle), whereas if the volume is increased the unstable mode consists of a nonaxisymmetric deformation. This kind of nonaxisymmetric deformation can also appear by decreasing the volume if the Bond number is large enough. A comparison with other previous partial theoretical analyses is presented, as well as with available experimental results.
\end{abstract}

\section{INTRODUCTION}

A liquid bridge is an idealization of the fluid configuration appearing in the crystal growth technique known as floating zone melting. The configuration analyzed consists of an isothermal, initially axisymmetric, mass of fluid held by surface tension forces between two parallel, coaxial, solid disks of the same diameter, as sketched in Fig. 1. The configuration is considered to be subject to a constant, axial gravity field. If the interface is assumed to be anchored to the sharp disk edges, the configuration can be uniquely characterized by the following dimensionless parameters: the slenderness, defined as the ratio of gap separation to disk diameter, $\Lambda=L /(2 R), L$ being the separation between the disks and $R$ being the common disk radius; the dimensionless volume of liquid, $V=V /\left(\pi R^{2} L\right)$, defined as the ratio of the physical volume $\mathbf{V}$ to the volume of a cylinder of the same $L$ and $R$; and the Bond number, Bo $=|\Delta \rho g| R^{2} / \sigma$, where $\Delta \rho$ is the difference between the liquid density and the surrounding medium density, $g$ the axial acceleration (positive when it has the direction of the $-z$ axis), and $\sigma$ the surface tension (both $\Delta \rho$ and $\sigma$ are assumed to be constant). Notice that modulus is used in the definition of Bo, because for equal disks the stability limits are the same no matter the sign of the effective axial mass force.

The availability of flight opportunities to carry out fluid and material sciences experiments in microgravity is giving rise to interest in the study of the behavior of liquid masses under microgravity conditions, the liquid bridge being one of the fluid configurations that is receiving increasing attention in those experiments. ${ }^{1-8}$ The mechanical aspects of the liquid bridge problem have received the attention of many investigators during the last two decades and, leaving apart dynamic effects, one can find in the literature a large number of papers dealing with the equi- librium shapes and the static stability limits of liquid bridges. A comprehensive review of these works has been done by Myshkis et al. ${ }^{9}$ Early studies dealt with liquid bridges between equal disks under gravitationless conditions, ${ }^{10-17}$ whereas the influence of Bond number has been the subject of more recent publications. ${ }^{18-26}$ The influence on stability limits of disks of different diameters has also been considered. ${ }^{17,26-29}$ Although the behavior at the stability limit is qualitatively similar no matter if the diameters are equal or slightly unequal, only liquid bridges between equal disks are considered in the following.

The problem of stability of liquid bridges under axial gravity has been investigated in some particular cases which are important for the floating zone melting technique. For cylindrical volumes $(V=1)$ the loss of stability with respect to axisymmetric perturbations has been studied by Coriell et al. ${ }^{19}$ and Meseguer. ${ }^{30}$ Analytical approximations for small Bond numbers have been calculated by Vega and Perales. ${ }^{20}$ The stability with respect to arbitrary (both axisymmetric and nonaxisymmetric) perturbations has been studied by Slobozhanin. ${ }^{21,22}$ He showed that for Bo $>3.06$ the nonaxisymmetric perturbations bound the stability region instead of the axisymmetric ones. In other works, the contact angle $\beta_{1}$ in the bottom disk or contact angle $\beta_{2}$ in the top disk is fixed, that is, a constant wetting angle $\pi-\beta_{1}$ at the bottom disk or $\beta_{2}$ at the top disk is considered. The case of a $90^{\circ}$ angle has been considered by Heywang ${ }^{31}$ and for arbitrary perturbations by Coriell and Cordes. ${ }^{18}$ In the works of Slobozhanin ${ }^{21,22}$ the values of the angle $90^{\circ}, 80^{\circ}$, and $75^{\circ}$ at each disk were investigated and it was shown that the particular value of the angle $\beta_{1}=90^{\circ}$ becomes a singular case as Bo tends to 0 .

The subject of the present investigation is the stability of a liquid bridge for arbitrary volumes, slendernesses, and Bond numbers. In the limiting case $B o=0$ (see Fig. 2), the 


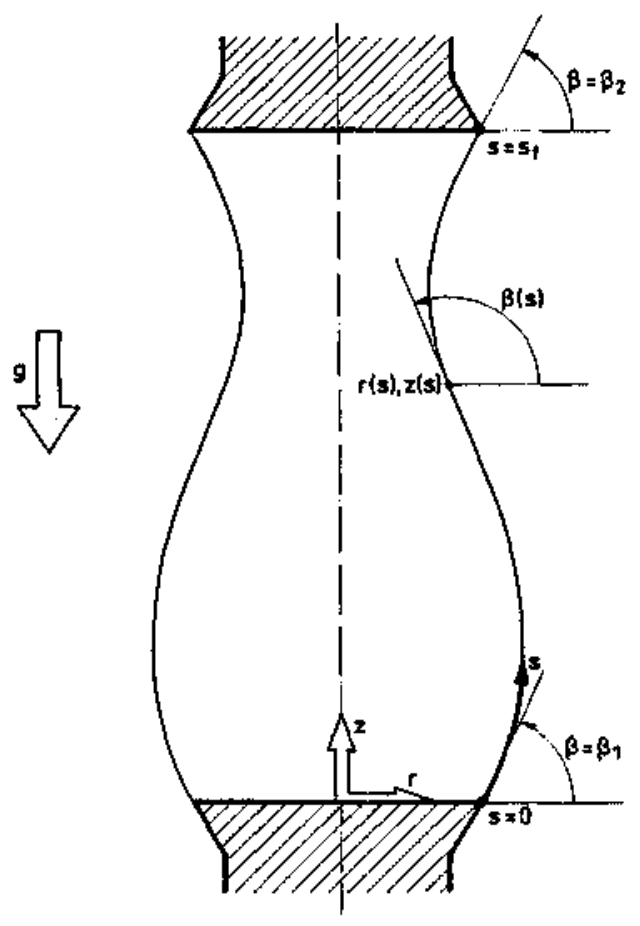

FIG. 1. Geometry and coordinate system for the liquid bridge problem.

region of stability with respect to axisymmetric perturbations has been constructed by Gillette and Dyson ${ }^{12}$ and Martínez, ${ }^{27}$ and with respect to arbitrary perturbations by Slobozhanin $^{14}$ (all the above-mentioned works of Slobozhanin were collected later in English ${ }^{9}$ ). According

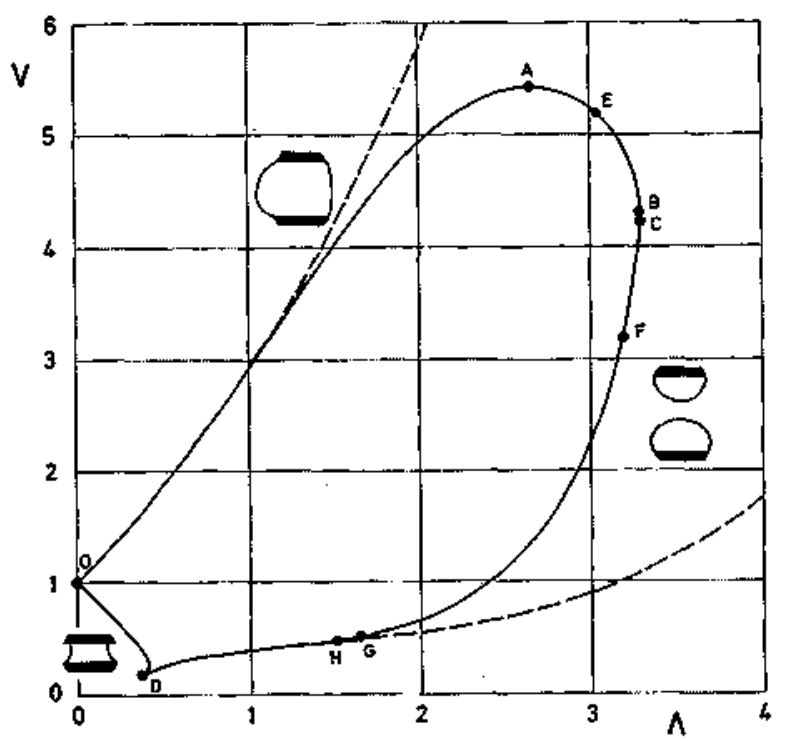

FIG. 2. Typical stability diagram of liquid bridges between equal disks subjected to a small constant axial acceleration [measured by Bond number: $B O=0.1$ (solid line), $B O=0$ (dashed line)]. The sketches indicate the different types of instability appearing on the different parts of the stability curve. A, maximum of volume; $B$, maximum of slenderness; $C$, transition between axisymmetric and nonaxisymmetric instabilities; $D$, zero angle at the top disk; $\mathbf{E}$, local minimum in pressure; $F$, local minimum of the angle at the top disk; $\mathrm{O}$, local maximum of the angle at the top disk; $\mathbf{H}$, local maximum of the pressure. to the last work, the boundary of stability region in $\Lambda \cdot V$ plane is unclosed and consists of three parts. On the upper boundary the bridge loses its stability with respect to nonaxisymmetric perturbations, on the right part of the lower boundary with respect to axisymmetric perturbations and on the left part of the lower boundary a detachment of wetting lines from the edges of disks appears. It was shown that for the case of zero wetting angle the latter part coincides with the boundary with respect to nonaxisymmetric perturbations and that in general the position of the left part of the lower boundary is determined by the value of the wetting angle.

The calculation of this boundary for nonzero wetting angles was carried out for the first time by Sanz and Martínez ${ }^{15}$ and more complete results can be found in Slobozhanin. ${ }^{32}$ The calculation of the upper boundary with respect to arbitrary perturbations and its experimental checking was also carried out by Russo and Steen. ${ }^{16}$

The influence of axial gravity on the character of deformation of the first and second parts of the boundary corresponding to $\mathrm{Bo}=0$ was determined by Barmin $e t a{ }^{23}$

According to the above-mentioned results, for each nonzero value of Bond number the stability diagram of capillary liquid bridges can be represented by a single closed piecewise curve on the $\Lambda-V$ plane. Liquid bridge configurations represented by points inside of the region bounded by this curve are stable whereas those lying outside are unstable. A typical stability limit curve for small Bond numbers (Bo=0.1) is shown in Fig. 2. In such a stability limit curve it is possible to distinguish three different parts. For volumes $V<1$ and small slendernesses, the instability is governed by the detachment of the interface (the wetting line) from the edges of the top disk (curve OD in Fig. 2 corresponds to zero wetting angle). Another part of the stability limit curve, corresponding to a minimum in the volume, is characterized by the axisymmetric breakage of the liquid bridge (curve DC where point $\mathrm{C}$ is always below the point of maximum slenderness, B), such a part of the stability curve being known in the literature as the minimum volume stability limit. Finally, the last part of the curve (curve OABC) is characterized by the loss of axisymmetry of the equilibrium shapes, i.e., a nonaxisymmetric deformation appears.

For rather small Bond numbers, the minimum volume stability limit with respect to axisymmetric perturbations has been determined by Meseguer and Sanz, ${ }^{24}$ Martínez et $a L .{ }^{25}$ and Perales $e t a l .^{26}$ The stability limits of minimum volume are well documented both theoretically and experimentally, and there are no relevant discrepancies in the results reported by different authors.

To our knowledge, apart from the mentioned work of Barmin et al., ${ }^{23}$ there is only one theoretical analysis dealing with the influence of Bond number on stability limits of maximum volume of general configurations (Martínez et $a l^{25}$ ). In Fig. 7 of that paper the stability regions for different values of the Bond number are shown. However, the stability curves were calculated by considering only axisymmetric perturbations, and, although the minimum volume stability limits presented there are applicable, the 
maximum volume stability limit shows discrepancies with experiments. For very short liquid columns (with slendernesses close to zero) only liquid bridges with volume very close to the cylindrical one can be stable in practice.

This paper aims to clarify the above-described situation, to determine the influence of Bond number on the stability limits of capillary liquid bridges, and to compare the theoretical results with the experimental ones which can be found in Perales et al. ${ }^{26}$ and Bezdenejnykh et al..$^{33}$ The boundary of the stability region with respect to arbitrary (both axisymmetric and nonaxisymmetric) perturbations is calculated and the transition point between these two types of perturbations is determined.

\section{MATHEMATICAL FORMULATION OF THE PROBLEM}

The geometry and notation used are shown in Fig. 1. It is assumed that the axial acceleration $g$ is directed downwards. The origin of the reference system is at the center of the lower disk and the $z$ axis points to the liquid. The arclength, $s$, of the meridian curve (outer shape) is measured from the point where $z=0$.

In the following, unless otherwise stated, all lengths are made dimensiontess with $L_{C}=\sqrt{\sigma /|\Delta \rho \cdot g|}$, a dimensionless radius $r_{0}=R / L_{C}$ and disk separation $h=L / L_{C}$, are defined. Axisymmetric equilibrium shapes of liquid bridges anchored to the sharp edges of the supporting disks are characterized by the dimensionless coordinates of the meridian curve as a function of the curve arclength, $r=r(s), z=z(s)$, which are functions of the configuration and stimuli applied, namely: slenderness $\Lambda$, liquid volume $V$; and residual acceleration in the axial direction of the column measured by the Bond number, Bo. Then, assuming the set of parameters $(\Lambda, V, \mathbf{B o})$ given, the equilibrium shape can be computed by numerically integrating the set of second-order differential equations:

$$
\begin{aligned}
& r^{\prime \prime}(s)=-z^{\prime}(s) \beta^{\prime}(s), \\
& z^{\prime \prime}(s)=r^{\prime}(s) \beta^{\prime}(s),
\end{aligned}
$$

where $\beta=\beta(s)$ is the angle between the tangent to the meridian of the equilibrium shape and the horizontal, and primes denote derivation with respect to arclength $s$. The angle $\beta$ can be computed from

$$
\beta^{\prime}(s)=\epsilon z(s)+C-\frac{z^{\prime}(s)}{r(s)} .
$$

In the last equation, $C$ is the dimensionless pressure difference between the reference pressure, $P$, inside the liquid bridge at $z=0$ and that of the surrounding medium, $P_{0}$,

$$
C=\frac{P-P_{0}}{\sqrt{|\Delta \rho \cdot g| \cdot \sigma}},
$$

and $\epsilon$ stands for the direction of the effective gravity

$$
\epsilon=-\operatorname{sign}(\Delta \rho \cdot g) \text {. }
$$

The set of differential equations must be solved with the boundary conditions ( $s_{f}$ is the final value of the arclength)

$$
\begin{aligned}
& r(0)=r_{0}=\sqrt{\mathrm{Bo}}, \\
& r\left(s_{f}\right)=r_{0}=\sqrt{\mathrm{Bo}}, \\
& z(0)=0, \\
& z\left(s_{f}\right)=h=2 \Lambda \sqrt{\mathrm{Bo}},
\end{aligned}
$$

and the unknown constant $C$ must be adjusted to fulfill the volume preservation condition

$$
V=\frac{\int_{0}^{s} f r^{2}(s) z^{\prime}(s) d s}{h r_{0}^{2}} .
$$

The problem can be solved as an initial-value problem by using a shooting method. The boundary conditions (4)(7) must be replaced by the initial conditions

$$
\begin{aligned}
& r(0)=r_{0}, \\
& r^{\prime}(0)=\cos \beta_{1}, \\
& z(0)=0, \\
& z^{\prime}(0)=\sin \beta_{1},
\end{aligned}
$$

and Eq. (3) can be integrated by using as initial condition

$$
\beta(0)=\beta_{1} ;
$$

$C, \beta_{1}$, and $s_{f}$ must be adjusted to fulfill the conditions (5), (7), and (8).

Following the method described by Myshkis et $a L^{9}$ to find the stability boundaries during the numerical integration of the system (1)-(3) with initial conditions (9)(13), the functions $\varphi_{01}(s), \varphi_{02}(s)$, and $\varphi_{1}(s)$ must be calculated from the differential equations

$$
\begin{aligned}
& -\varphi_{01}^{\prime \prime}(s)-\frac{r^{\prime}(s)}{r(s)} \varphi_{01}^{\prime}(s)+a(s) \varphi_{01}(s)=0, \\
& -\varphi_{02}^{\prime \prime}(s)-\frac{r^{\prime}(s)}{r(s)} \varphi_{02}^{\prime}(s)+a(s) \varphi_{02}(s)=0 \\
& -\varphi_{1}^{\prime \prime}(s)-\frac{r^{\prime}(s)}{r(s)} \varphi_{1}^{\prime}(s)+\left(a(s)+\frac{1}{r^{2}(s)}\right) \varphi_{1}(s)=0,
\end{aligned}
$$

with

$$
a(s) \equiv \epsilon r^{\prime}(s)-\beta^{\prime 2}(s)-\left(\frac{z^{\prime}(s)}{r(s)}\right)^{2}
$$

and the initial conditions

$$
\begin{array}{ll}
\varphi_{01}(0)=0, & \varphi_{01}^{\prime}(0)=1, \\
\varphi_{02}(0)=1, & \varphi_{02}^{\prime}(0)=0, \\
\varphi_{1}(0)=0, & \varphi_{1}^{\prime}(0)=1 .
\end{array}
$$

Besides, the following function is to be calculated during the integration process: 


$$
\begin{aligned}
\mathscr{D}(s)= & \varphi_{01}(s)\left(\cos \beta_{1} \int_{0}^{s} r(s) \varphi_{02}(s) d s-\frac{1}{2}\left[r^{2}(s)-r_{0}^{2}\right]\right) \\
& +\left[r^{\prime}(s)-\varphi_{02}(s) \cos \beta_{1}\right] \int_{0}^{s} r(s) \varphi_{01}(s) d s .
\end{aligned}
$$

Depending on which function, $\mathscr{D}(s)$ or $\varphi_{1}(s)$ vanishes first during the integration, the perturbation which destabilizes the axisymmetric equilibrium state is axisymmetric or nonaxisymmetric, respectively.

According to the election of the reference system, in the following the value $\epsilon=-1$ is assumed. By integrating Eq. (2) taking into account Eq. (3) the following useful expression, valid for any liquid bridge between equal disks is obtained:

$$
\sin \beta_{2}=\sin \beta_{1}+\Lambda \mathrm{Bo}(V-1) .
$$

\section{NUMERICAL ALGORITHM}

For integrating the systems of equations (1)-(3) and (14)-(16) a Runge-Kutta method with a constant advancing step $\delta$ has been used. Let us fix a value of $r_{0}$, i.e., of $B o$, and assume a value for $\beta_{1}$ (say $\beta_{1}=\dot{\beta}_{l}^{(1)}$ ) in the interval $-\pi / 2<\beta_{1}^{(1)}<\pi$. For this value $\beta_{1}^{(1)}$ a value for $C=C_{g}$ is guessed. For given values of $\beta_{1}=\beta_{1}^{(1)}$ and $C=C_{g}$ the integration of Eqs. (1)-(3) and (14)-(16) with initial conditions (9)-(13) and (18), and the calculation of the function $\mathscr{D}(s)$, are carried out. The integration process continues until the point $s=s^{*}$ for which either of the functions $\mathscr{D}(s)$ or $\dot{\varphi}_{1}(s)$ becomes equal to zero for the first time after the initial point $s=0$.

During the integration, the value $r\left(s^{*}\right)$ is calculated. The condition

$$
\left|r\left(s^{*}\right)-r_{0}\right|<l
$$

is controlled (usually a value $l=\delta$ is taken). If this condition is not satisfied and $r\left(s^{*} ; r_{0}, \beta_{1}^{(1)}, C_{g}\right)>r_{0}$, then it is necessary to choose a value $C>C_{8}$ for the next iteration (with the same value of $\beta_{1}=\beta_{1}^{(1)}$ ). The iteration process is continued until a value $C=C_{g}$ for which $r\left(s^{*}, r_{0}, \beta_{1}^{(1)}, C_{g}\right)<r_{0}$ is found. On the contrary if $r\left(s^{*} ; r_{0}, \beta_{1}^{(1)}, C_{g}\right)<r_{0}$ then it is necessary to decrease the value of $C$ with respect to $C_{z}$

In this way, an interval of values of the parameter $C$ with their extremes having different signs of $r\left(s^{*}\right)-r_{0}$ is determined. Then a value $C=C^{*}$ for which condition (21) is satisfied can be determined by a bisection root finder. The corresponding equilibrium shape $\left(C=C^{*}, 0<s<s^{*}\right)$ is the critical one (the one corresponding to the stability limit) for the given $\mathrm{Bo}=r_{0}^{2}$ and $\beta_{1}=\beta_{1}^{(1)}$.

For each critical equilibrium shape the following values are monitored:

$$
\begin{array}{ll}
r_{0}, \quad \beta_{1}\left(=\beta_{1}^{(1)}\right), \quad C=C^{*}, & h=z\left(s^{*}\right), \\
\beta_{2}\left(=\beta\left(s^{*}\right)\right), \quad \Lambda=h / 2 r_{0}, & V\left(s^{*}\right) .
\end{array}
$$

It must also be distinguished which of the two functions $\mathscr{D}(s)$ and $\varphi_{1}(s)$ changes the sign at the point $s=s^{*}$ (loss of stability with respect to axisymmetric or nonaxisymmetric perturbations).
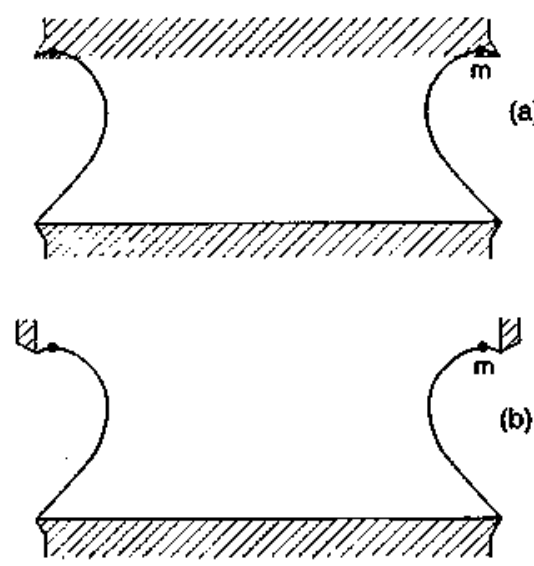

FIG. 3. Sketch of the situation appearing when the limiting cquilibrium shape has a negative value of the angle in the top disk, $\beta_{2}<0$; (a) when a solid disk is considered, (b) when a tube is used as the upper support of the liquid column.

The above values $\Lambda$ and $V\left(s^{*}\right)$ determine a point of the stability boundary in the $(\Lambda, V)$ map. To find another point of this boundary it is necessary to change the value of $\beta_{1}$ (say $\beta_{1}=\beta_{1}^{(2)}$ ) and repeat the whole procedure. By such a way the whole boundary is constructed for a given value of Bo.

If $\beta\left(s^{*}\right)<0$, the critical profile contains a point $m$ [see Fig. 3(a)], for which

$$
r^{\prime}\left(s_{m}\right)=1, \quad z^{\prime \prime}\left(s_{m}\right)<0 \quad \text { (with } 0<s_{m}<s^{*} \text { ). }
$$

Such profile cannot be realized between flat solid disks although it could be obtained if a tube were used as upper support [see Fig. 3(b)]. In this case of edge detachment it is necessary to find the shape of the limiting profile for given $B o$ and $\beta_{1}$ as well. In the results presented below it has been assumed that the liquid has a perfect wetting, i.e., that any angles $\beta_{1}<\pi, \beta_{2}>0$ are possible. The limiting profile $\left(0<s<s_{m}\right)$ is characterized by the equality $r\left(s_{m}\right)=r_{0}$. The limiting profile is obtained by an analog iteration procedure on the parameter $C$ until the condition

$$
\left|r\left(s_{m}\right)-r_{0}\right|<l \text {. }
$$

is satisfied. Nevertheless, by using Eq. (20), it can be deduced that, if $0<\beta_{1}<\pi$ and $V>1$ then $\beta_{2}>0$, and thus the above-described situation cannot be reached. Slobozhanin ${ }^{21,22}$ (see also Myshkis et al. ${ }^{9}$ ) also deduced that $\beta_{2}>0$ for the critical profile if $-\pi / 2<\beta_{1}<0$.

\section{RESULTS AND ANALYSIS}

Following the above-described method, the stable region in the $\Lambda-V$ diagram has been computed for different Bond numbers in the range $0.005<\mathrm{Bo}<7.0$. The results have been plotted in Fig. 4 assuming that the liquid bridge is held between solid disks, that is, wherever necessary, the stability limit has been substituted by the curve for $\beta_{2}=0$. Besides these stability limits for nonzero Bond numbers, the distinguished case $B O=0$ has also been plotted after Slobozhanin. ${ }^{14}$ 


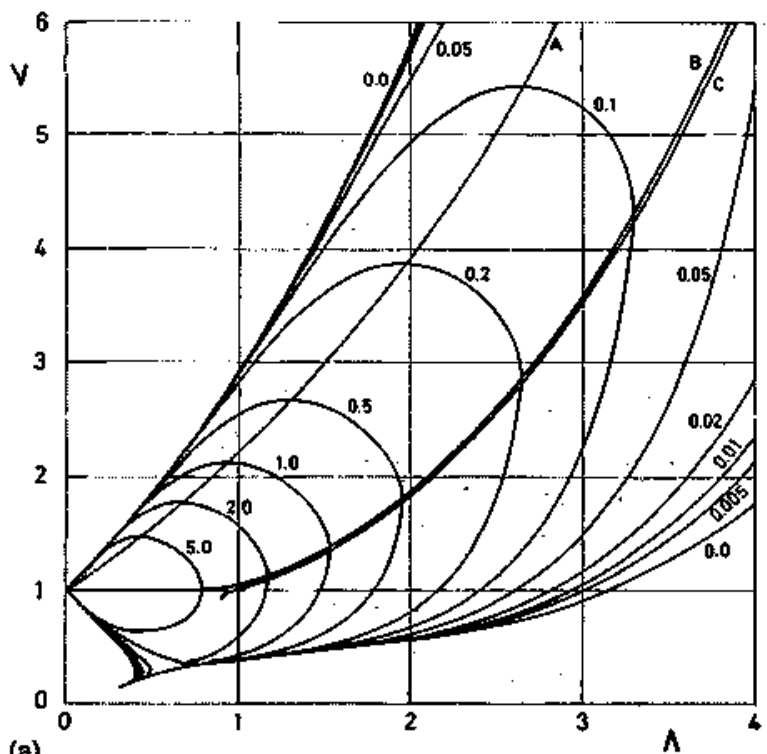

(a)

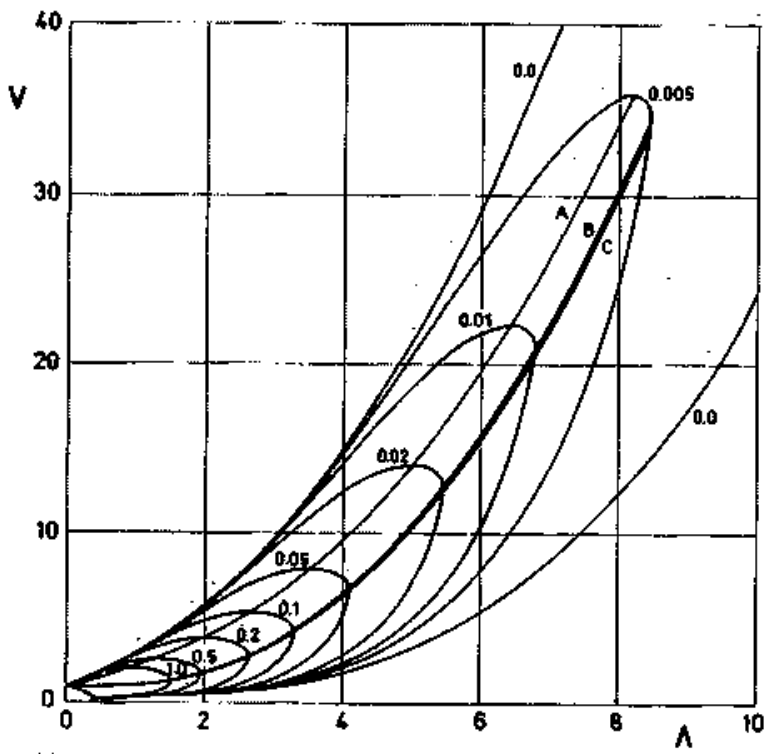

(b)

FIG. 4. Influence of the Bond number on the stability limits of liquid bridges between equal disks. Numbers on the curves indicate the value of the Bond number. Curve labeled $A$ shows the locus of the points with maximum volume for a given value of Bo, curve $B$ shows the locus of the points with maximum slenderness for a given value of $B$, and curve $C$ the transition between axisymmetrical breakage and nonaxisymmetric deformation.

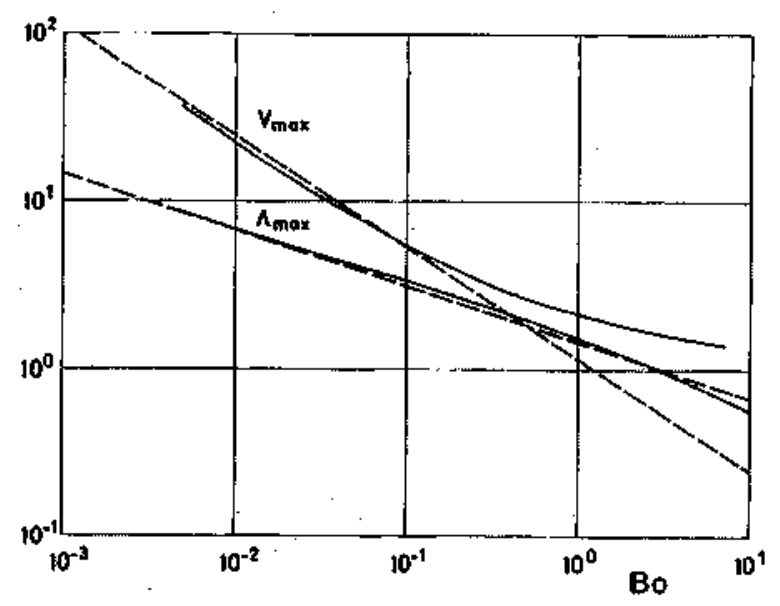

FIG. 5. Maximum stable slenderness (for any volume) and maximum stable volume as a function of Bond number. Dashed lines correspond to asymptotic approximations given by Eqs. (22) and (23).

The calculations show that in the part of the boundary which bounds the largest possible volume for a given slenderness and Bond number (Fig. 2, curve OAB), the loss of stability is always due to nonaxisymmetric perturbations, i.e., function $\varphi_{\mathrm{I}}(s)$ has its smallest zero for a smaller arclength than $\mathscr{D}(s)$.

One of the characteristic points of this segment of the boundary is the point where the maximum possible volume, $V_{\max }$, for a given Bond number is obtained (Fig. 2, point $\mathrm{A}$ ). The locus of these points has been determined (curve A on Fig. 4). The values of the relevant parameters for this curve have been collected, for different values of the Bond number, in Table I. On curve A it can be proved that the minimum value of $\beta_{1}$ for each Bond number is reached.

The value of $V_{\max }$ as a function of Bond number has been plotted in Fig. 5 . It can be observed that for Bo $<1$, $V_{\max }$ behaves as $\mathrm{Bo}^{-2 / 3}$.

The analytical approximation

$$
V_{\max }=\left(\frac{2}{3}\right)^{1 / 3} \mathrm{Bo}^{-2 / 3}
$$

deduced after Myshkis et al. ${ }^{9}$ (Sec. 2.4.3) by assuming the shape of a pendant drop has also been plotted. The error of this approximation is smaller than $10 \%$ for Bo<0.1.

TABLE I. Values of the parameters for the maximum possible volume, $V_{\max }$, for different Bond numbers (point $\mathbf{A}$ in Fig. 2).

\begin{tabular}{|c|c|c|c|c|c|}
\hline Bo & $\Lambda$ & $\boldsymbol{V}_{\text {Fах }}$ & $\beta_{1}$ & $\beta_{2}$ & $C$ \\
\hline 0.01 & 6.44 & 22.338 & -24.4 & 105.8 & 3.86 \\
\hline 0.05 & 3.53 & 7.977 & -18.9 & 115.1 & 3.20 \\
\hline 0.10 & 2.65 & 5.426 & -17.2 & 118.8 & 2.97 \\
\hline 0.20 & 1.96 & 3.865 & -16.0 & 121.9 & 2.77 \\
\hline 2.00 & 0.67 & 1.770 & -13.8 & 127.5 & 2.24 \\
\hline 5.00 & 0.43 & 1.474 & -13.5 & 128.3 & 2.11 \\
\hline 7.00 & 0.36 & 1.398 & -13.4 & 128.5 & 2.07 \\
\hline
\end{tabular}


TABLE II. Values of the parameters for the maximum possible slenderness, $\Lambda_{\max }$, for different Bond numbers (point $B$ in Fig. 2 ).

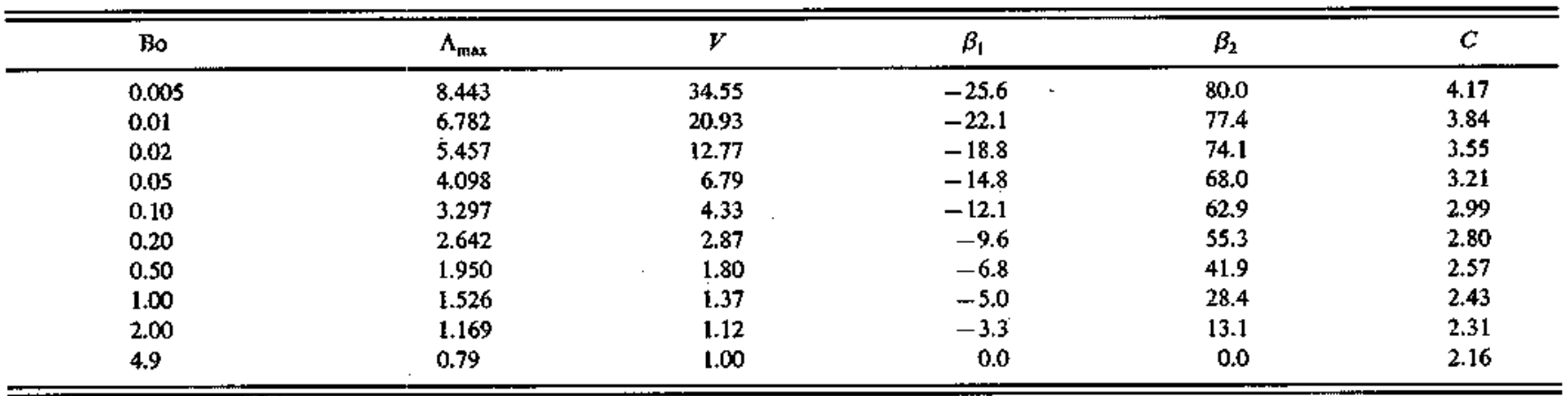

The second characteristic point in the maximum volume stability limit is the point where the maximum length, $\Lambda_{\max }$, for a given Bond number is achieved (point B in Fig. 2). The locus of these points has been plotted in Fig. 4 (curve B) and their relevant parameters have been listed in Table II. The dependence on Bond number of the maximum slenderness (not necessarily for $V=1$ ) has been plotted in Fig. 5. For Bo<1 $\Lambda_{\max }$ behaves as Bo ${ }^{-1 / 3}$. The asymptotic expression

$$
\Lambda_{\max }=\sqrt[3]{3} \mathrm{Bo}^{-i / 3}
$$

was suggested by Langbein ${ }^{34}$ who considered only axisymmetric perturbations of the equilibrium state. The comparison of numerical results with the above-mentioned asymptotic expression (see Fig. 5) shows that they agree almost perfectly (the error is smaller than $6 \%$ for Bo<0.1). Although for point $B$ the destabilizing perturbations are always nonaxisymmetric, the agreement can be explained by the fact that point $B$ is placed very close to the transition between axisymmetric and nonaxisymmetric destabilization for $\mathrm{Bo}<3.06$.

Curve B coincides with $V=1$ for Bo>4.9 $(\Lambda<0.79)$. It was shown by Slobozhanin ${ }^{21,22}$ that critical profiles for $V=1$ and $B o>3.0618 \quad(\Lambda<0.9763)$ have the property $\beta_{1}=\beta_{2}=0$. Besides, $\beta_{2}>0$ for any critical profile with $V>1$. Consequently, the point where $\beta_{2}=0$ (point $\mathrm{D}$ of Fig. 2) lies on the line $V=1$ for Bo>3.0618 $(\Lambda<0.9763)$ and thus, for $B o>4.9$ point $D$ coincides with point $B$. Taking into account the importance of the dependence of the maximum slenderness for cylindrical volumes (it coincides with curve $B$ for $B o>4.9$ and with the locus of point $D$ for Bo $>3.0618$ ), this limit has been plotted in Fig. 6 based in numerical results of Slobozhanin. ${ }^{21}$

Point C (see Fig. 2) separates the part of the boundary where the loss of stability is due to axisymmetric perturbations from where it is due to nonaxisymmetric ones. Curve $\mathrm{C}$ corresponding to these points has been plotted in Fig. 4 and the values of their parameters have been collected in Table III. Curve $C$ crosses $V=1$ for $\Lambda=0.9763$ which coincides with the results obtained for $V=1$ by Slobozhanin. ${ }^{21,22}$

Curve $\mathrm{C}$ can be continued in the region $V<1$ only if the constraint $\beta_{2}>0$ is relaxed and a configuration like the one sketched in Fig. 3(b) is permitted. A detail of the region has been plotted in Fig. 7. Nevertheless the value of $\beta_{2}$ is negative in the stability boundaries plotted for $V<1$ and should be replaced by the lines $\beta_{2}=0$ if solid disks [see Fig. 3(a)] were considered. Curve $C$ is tangent to the stability limit for $B O=3.595$ and thus, for larger values of $B \circ$ there is no transition and all breakages or deformations beyond the stability limit are nonaxisymmetric.

It is interesting to pinpoint the behavior of the solution in the vicinity of point $\mathrm{C}$. A typical situation is represented in Fig. $8(\mathrm{Bo}=0.05)$. In the $\Lambda-V$ plane, close to the neighborhood of point C, the line 1-C-B-2 [Fig. 8(a)] determines the loss of stability with respect to nonaxisymmetric perturbations $\left[\varphi_{1}\left(s^{*}\right)=0\right]$ and the line 3-C-4 with respect to axisymmetric perturbations $\left[\mathscr{P}\left(s^{*}\right)=0\right]$. These lines touch each other in point $C$. The angle of inclination of the common tangent with respect to the $\Lambda$ axis is acute (it means that in point $B$ the loss of stability occurs with respect to nonaxisymmetric perturbations). It could seem that by considering arbitrary perturbations the stability will always be lost on the section 1-C-B-2, i.e., with respect to nonaxisymmetric perturbations. Nevertheless, the analysis shows that along the same integral curve of the problem (1)-(3), (9)-(13) the zero of function $\mathscr{D}(s)$ appears earlier (for smaller $\left.s^{*}\right)$ than the zero of the function $\varphi_{1}(s)$ if $V<V_{C}$ and later if $V>V_{C}$ see Fig. 8(b). Thus, the boundary is determined by curve 3-C if $V<V_{C}$ and by curve C-B-2 if $V>V_{C}$

The lines $\beta_{1}=$ const and $\beta_{2}=$ const have been plotted in the $\Lambda-V$ plane in Figs. 9 and 10 , respectively. The points of crossing of the lines $\beta_{1}=$ const with the stability boundaries allow one to solve the problem of bridge stability under a fixed value of $\beta_{1}$ for different values of Bond number. The

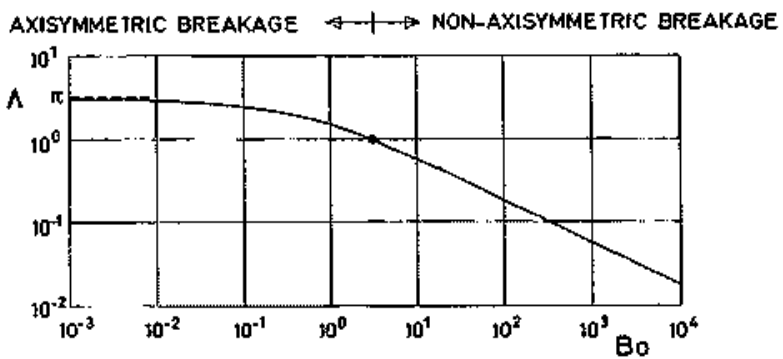

FIG. 6. Maximum stable slenderness for cylindrical volume $(V=1)$ as a function of Bond number (after Slobozhanin ${ }^{21}$ ). 
TABLE III. Values of the parameters at the point where the stability lossing changes from that due to axisymmetric perturbations to that due to nonaxisymmetric perturbations (point $\mathrm{C}$ in Fig. 2).

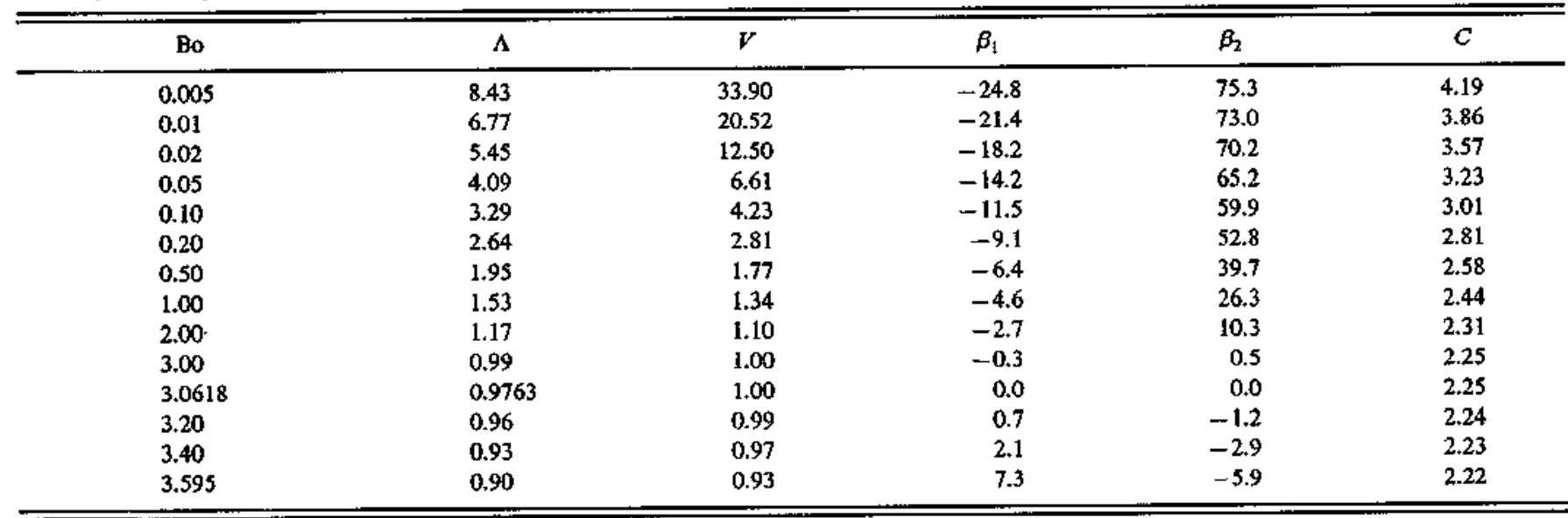

lines $\beta_{2}=$ const may be used for solving the analog problem of a given value of $\beta_{2}$. The two above problems are of interest in the study of the stability of a melting zone. The comparison of the obtained results with the stability investigation for $\beta_{1}=90^{\circ}, 80^{\circ}, 75^{\circ}$ and $\beta_{2}=90^{\circ}, 100^{\circ}, 105^{\circ}$ which was carried out earlier by Slobozhanin ${ }^{21,22}$ (see also Myshkis et $a L^{9}$ ) leads to a complete agreement.

The relationship (20) is useful for analysis of behavior of these lines. In particular, it is deduced that $\beta_{1}=\beta_{2}$ on the straight line $V=1$. The behavior of $\beta_{1}$ along the stability limit for a given Bond number is quite simple. In point $O$ (see Fig. 2) $\beta_{1}=0$, its value decreasing with increasing volume up to a minimum which coincides with the point

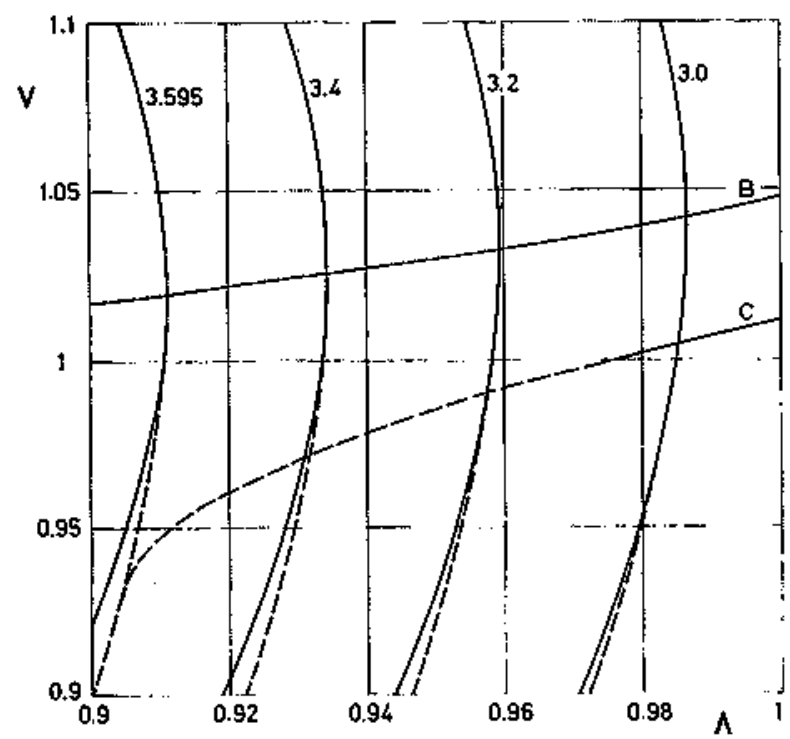

FIG. 7. Detail of the stability limit in the neighborhood of the point where the transition between axisymmetric to nonaxisymmetric breakages (curve $C$ ) happens for $V=1$. Curve $C$ has been dashed in the points where $\beta_{2}<0$. Curve $B$ is the locus of points with maximum slenderness. The stability limits (nonaxisymmetric instability if $V>V_{C}$ or axisymmetric instability if $V<V_{C}$ ) have been dashed if $\beta_{2}<0$ and substituted by the limiting shapes with $\beta_{2}=0$. Numbers on the curves indicate the value of Bond number, Bo.
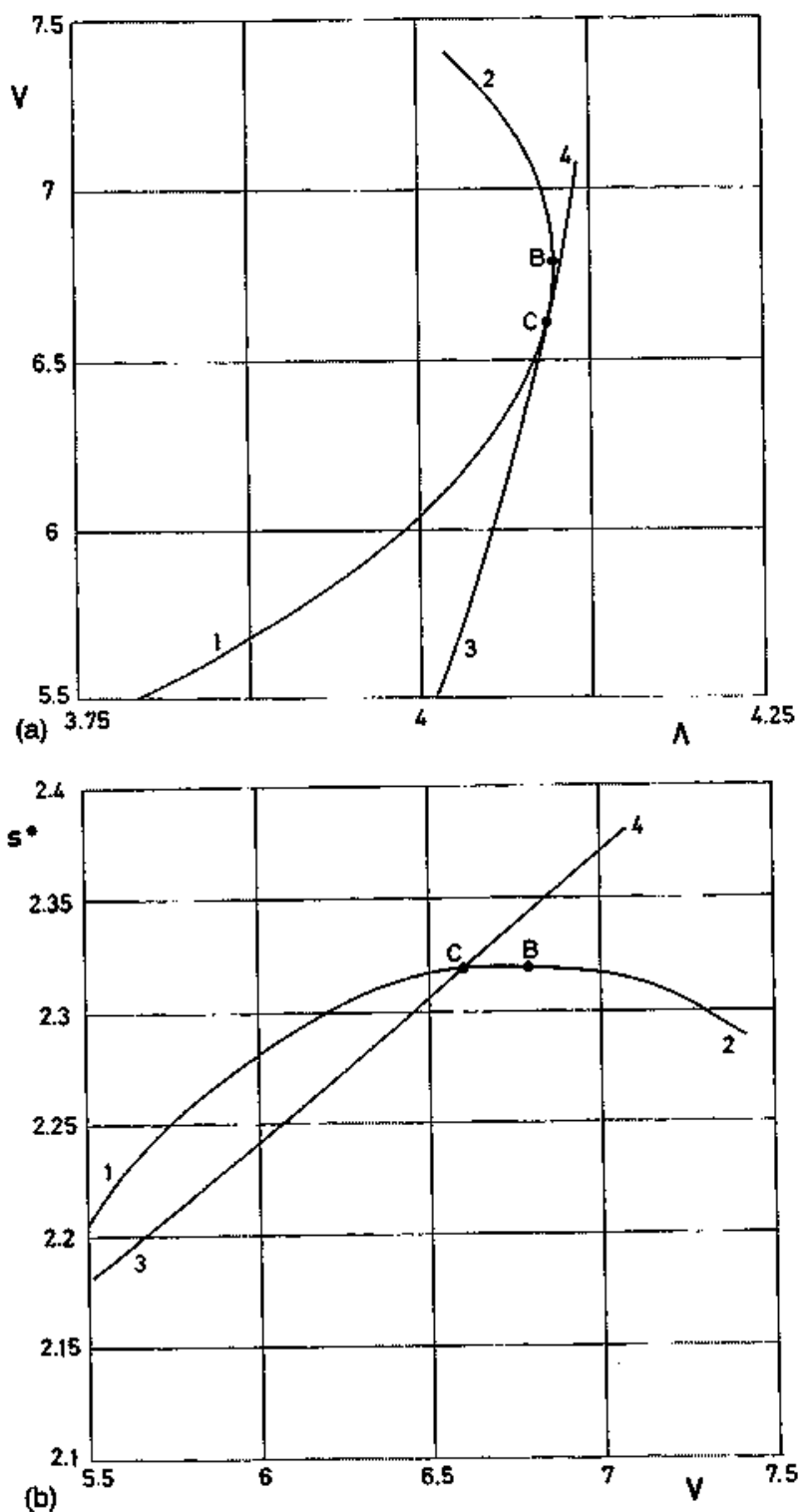

FIG. 8. Transition between axisymmetric and nonaxisymmetric modes of instability for $B=0.05$. (a) In the $A-V$ plane. (b) Arclength of the critical profile as a function of volume. Point $B$ corresponds to maximum length and point $\mathrm{C}$ to the transition between axisymmetric and nonaxisymmetric instabilities. 


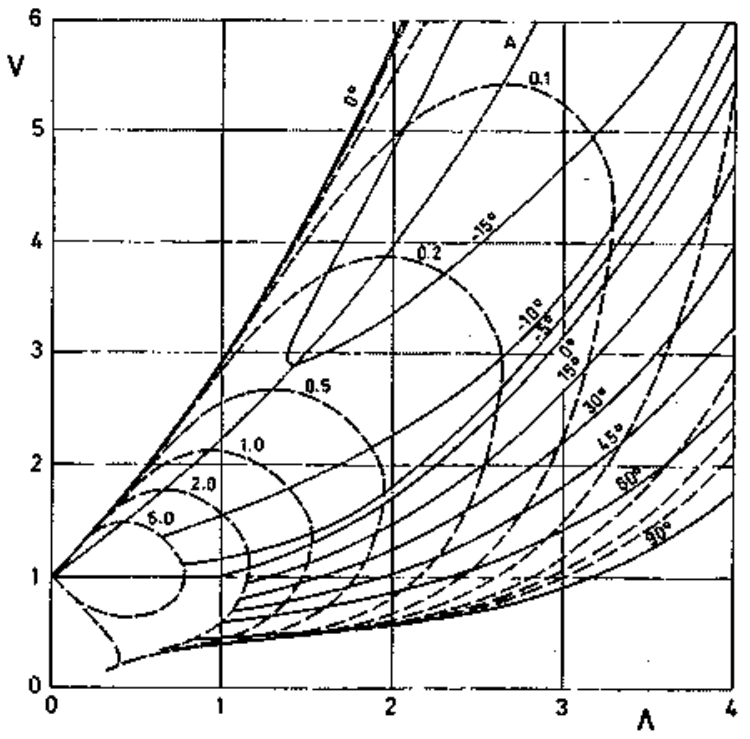

FIG. 9. Values of the angle in the bottom disk $\beta_{1}$ at the stability limit. Numbers on the dashed curves indicate the value of the Bond number. Curve $A$ is the locus of minimum value of $\beta_{1}$ for each Bond number.

for maximum volume (point $A$, for values of $\beta_{l_{\text {min }}}$ see values of $\beta_{1}$ in Table $I$ ), and then increasing monotonically up to point $D$. If a nonperfect wetting were considered there would be an upper limit for $\beta_{1}$. Remarkable values are $\beta_{1}=\pi / 2$, which coincides with the minimum volume stability boundary for $\mathrm{Bo}=0, \Lambda>2.13$ (see Myshkis et $a l^{9}$ ) and $\beta_{1}=0$, which coincides with the maximum volume stability boundary for $\mathrm{Bo}=0$.

The behavior of $\beta_{2}$ is quite different. For a fixed Bond number the value for point $O$ is $\pi$, it decreases up to a local minimum (point $F$ in Fig. 2 and curve $F$ in Fig. 10),

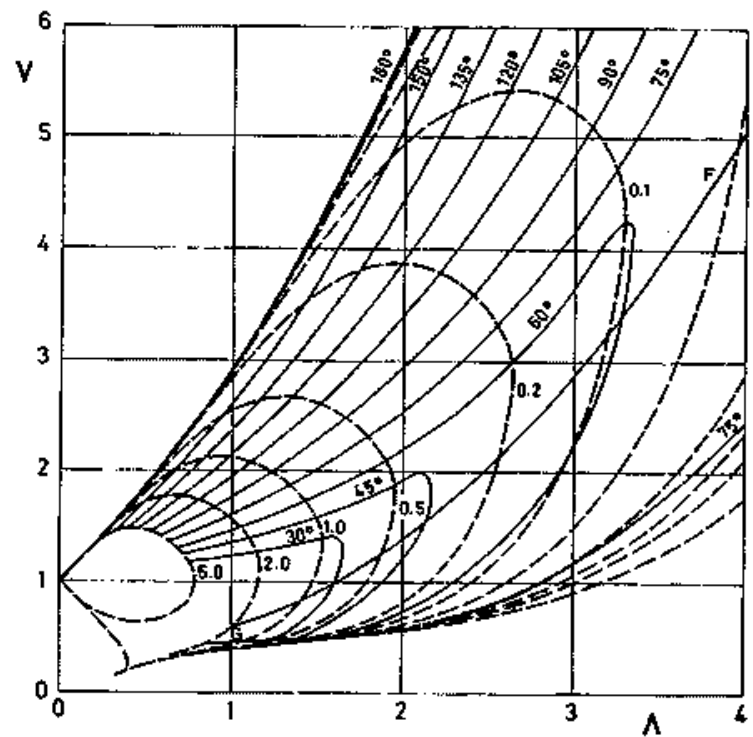

FIG. 10. Values of the angle in the top disk $\beta_{2}$ at the stability limit. Numbers on the dashed curves indicate the value of the Bond number. Curve $F$ is the locus of the local minimum of $\beta_{2}$ for each Bond number and curve $G$ that of the local maximum.
TABLE IV. Values of the parameters in the point of the stability boundary where $\beta_{2}=0$ (point $\mathrm{D}$ in Fig. 2).

\begin{tabular}{ccccc}
\hline \hline Bo & $\Lambda$ & $V$ & $\beta_{1}$ & $C$ \\
\hline 0.005 & 0.361 & 0.165 & 179.9 & -6.26 \\
0.01 & 0.362 & 0.165 & 179.8 & -4.39 \\
0.02 & 0.363 & 0.166 & 179.7 & -3.06 \\
0.05 & 0.365 & 0.166 & 179.1 & -1.84 \\
0.1 & 0.368 & 0.170 & 178.2 & -1.19 \\
0.2 & 0.376 & 0.178 & 176.5 & -0.67 \\
0.5 & 0.401 & 0.197 & 170.7 & -0.09 \\
1.0 & 0.450 & 0.231 & 159.8 & 0.41 \\
2.0 & 0.629 & 0.329 & 122.4 & 1.36 \\
\hline \hline
\end{tabular}

increases to a local maximum (curve $G$ ), and finally decreases up to the point where $\beta_{2}=0$ (point $D$ ). The parameters for this limiting situation when solid disks are considered have been collected in Table IV. It must be pointed that there are no positive extremes for $\beta_{2}$ if $\mathrm{Bo}>3$. Remarkable values are $\beta_{2}=\pi / 2$, which coincides with the minimum volume stability boundary for $B=0, \Lambda>2.13$ (see Myshkis et $a l^{9}$ ) and $\beta_{2}=\pi$, which coincides with the maximum volume stability boundary for $\mathrm{Bo}=0$ and any length.

To help in further calculations, the lines $C=$ const in the stability limit have been plotted in Fig. 11 and the local extremes of $\beta_{1}, \beta_{2}$, and $C$ have been collected in Table $\mathrm{V}$. The dimensionless pressure difference reaches a local minimum (curve E) and a local maximum (curve $\mathrm{H}$ ).

The analysis of stability when arbitrary (both axisymmetric and nonaxisymmetric) perturbations are taken into account leads to a shortening of the stability region for a given Bond number compared with the analysis of stability with respect to axisymmetric perturbations only. This fact explains the difference between present results and those

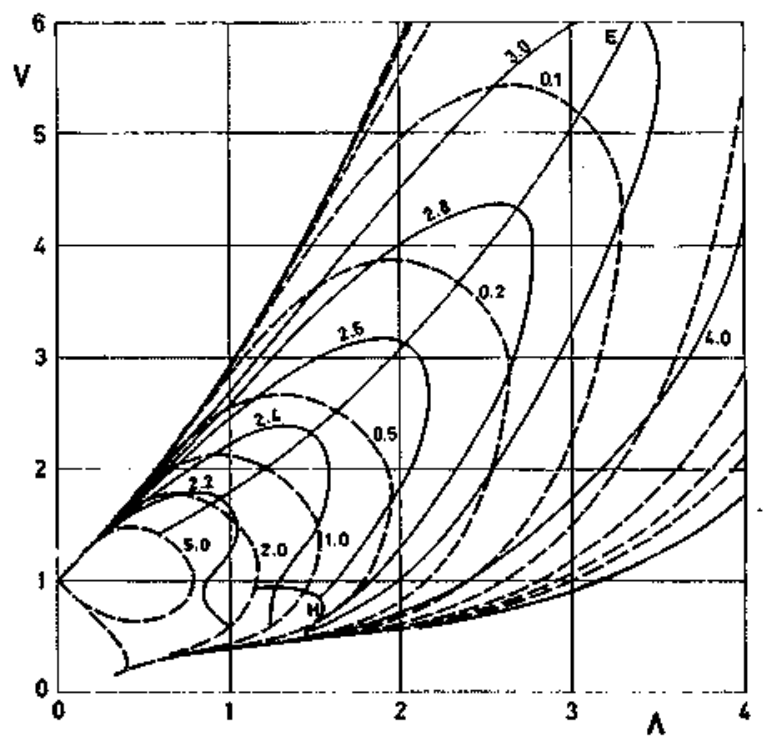

FIG. I1. Values of the constant $C$ at the stability limit. Numbers on the dashed curves indicate the value of the Bond number. Curve $E$ is the locus of the local minimum of $C$ for each Bond number and curve $H$ that of the local unaximum. 
TABLE V. Local extremes of $\beta_{1}, \beta_{2}$ and $C$ for fixed Bond number on the stability boundary.

\begin{tabular}{lccccc}
\hline \hline Bo & $\beta_{1 \min }$ & $\beta_{2 \max }$ & $\beta_{2 \min }$ & $C_{\max }$ & $C_{\min }$ \\
\hline 0.005 & -27.24 & 84.67 & 74.46 & 20.837 & 4.141 \\
0.01 & -24.36 & 82.49 & 72.43 & 14.807 & 3.803 \\
0.02 & -21.78 & 79.42 & 69.74 & 10.558 & 3.501 \\
0.05 & -18.92 & 73.51 & 64.78 & 6.820 & 3.149 \\
0.1 & -17.24 & 67.13 & 59.47 & 4.971 & 2.914 \\
0.2 & -15.96 & 58.42 & 52.31 & 3.721 & 2.702 \\
0.5 & -14.79 & 42.30 & 38.66 & 2.778 & 2.458 \\
1.0 & -14.21 & 25.48 & 23.71 & 2.495 & 2.301 \\
2.0 & -13.81 & 3.67 & 2.91 & 2.334 & 2.170 \\
5.0 & -13.49 & & & 2.166 & 2.033 \\
\hline \hline
\end{tabular}

obtained by Martínez et $a L^{25}$ considering only axisymmetric perturbations, both of which have been plotted in Fig. 12. Although in the region corresponding to axisymmetric breakages the results match perfectly (as it happens when compared with the results of Perales et al. ${ }^{26}$ for the minimum volume stability limit), they are different for the maximum volume stability limit (for $\Lambda \rightarrow 0$ when only axisymmetric perturbations are considered $V \rightarrow \pi$, while if arbitrary perturbations are considered $V \rightarrow 1$ ).

The present results have been compared with the experimental results reported by Bezdenejnykh et al. . $^{33}$ (Fig. 13). The qualitative agreement is excellent but it is difficult to obtain quantitative agreement. The authors used a nominal value for surface tension of $\sigma=0.072 \mathrm{~N} \mathrm{~m}^{-1}$ to calculate the Bond number, which seems to be rather optimistic (water is easily contaminated and the surface tension decreases significantly with contamination). A good agreement might be obtained if a lower value for surface tension (approximately $\sigma=0.06 \mathrm{~N} \mathrm{~m}^{-1}$ ) had been used in reducing experimental results.

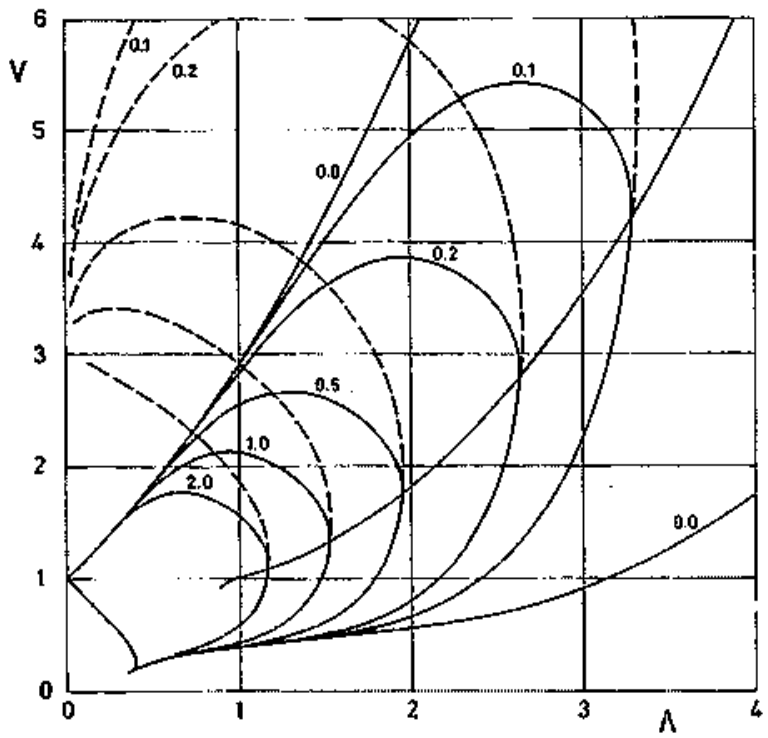

FIG. 12. Influence of the Bond number on the stability limits of liquid bridges between equal disks according to Martínez et al. ${ }^{25}$ (dashed lines) and according to the present method. Numbers on the curves indicate the value of the Bond number.

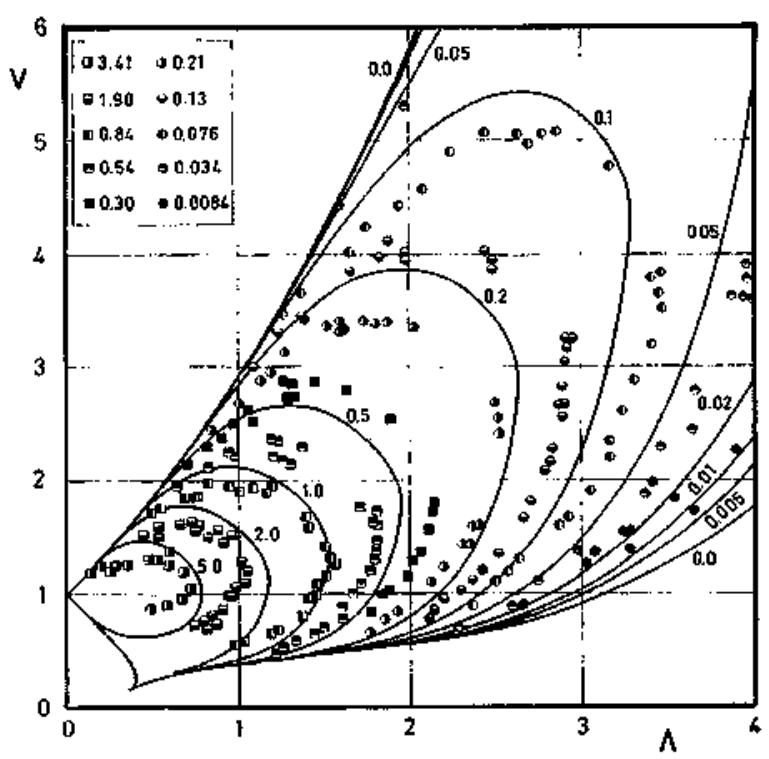

FIG. 13. Comparison with the experimental stability limits of liquid bridges between equal disks reported by Bezdenejnykh $e t a l^{33}$ The symbols indicate experimental results for the Bond numbers quoted in the legend. Numbers on the curves indicate the value of Bond number, Bo.

Finally, note that the loss of stability with respect to nonaxisymmetric perturbations may lead either to establish a new stable liquid bridge which has a nonaxisymmetrical shape or to the breakage of the bridge. This question has not been theoretically solved yet.

\section{CONCLUSIONS}

The stability region for arbitrary Bond numbers of liquid bridges spanning equal disks have been obtained. Three different kinds of instability can be observed; namely, axisymmetric breakage, nonaxisymmetric deformation, and interface detachment from the disk edge.

The behavior of the stability limit has been analyzed in detail in the regions where an extreme in any parameter appears, and the point where the transition between axisymmetric, and nonaxisymmetric destabilization occurs has been determined.

The results obtained have been compared with other existing theoretical and experimental results, the agreement being good.

\section{ACKNOWLEDGMENTS}

The authors are indebted to M. Gómez for her help in carrying out the numerical computations.

This work has been partly sponsored by the Spanish Comisión Interministerial de Ciencia y Tecnología (CICYT) and is part of a more general endeavor for the study of fluid physics and materials processing under microgravity (Project No. ESP92-0001-CP).

'I. Martínez, "Liquid column stability," in ESA SP-222 (Europears Space Agency, Parts, 1984), p. 31.

${ }^{2}$ I. Martínez and A. Eyer, "Liquid bridge analysis of silicon crystal growth experiments under microgravity," J. Cryst. Growth 75, 535 (1986). 
${ }^{3}$ I. Martínez and A. Sanz, "Long liquid bridges aboard sounding rockets," ESA J. 9, 323 (1985).

4J. Meseguer, A. Sanz, and J. López, "Liquid bridge breakages aboard Spacelab-D1," J. Cryst. Growth 78, 325 (1986).

${ }^{5}$ I. Martínez, "Stability of liquid bridges. Results of SL-D1 experiments," Acta Astronaut. 15, 449 (1987).

'I. Martínez, "Stability of long liquid columns in Spacelab-D1," in ESA SP-256 (European Space Agency, Paris, 1987), p. 235.

${ }^{7}$ I. Martinez and J. Meseguer, "Floating liquid zones in microgravity," in Scientific Results of the German Spacelab Mission D1, edited by P. R. Sahm, R. Jansen, and M. H. Keller (DFVLR, Köln, 1987), p. 105.

${ }^{8}$ I. Martínez, A. Sanz, J. M. Perales, and J. Meseguer, "Freezing of a long liquid column on the Texus-18 sounding-rocket flight," ESA J. 12, 483 (1988).

${ }^{9}$ A. D. Myshkis, V. G. Babskii, N. D. Kopachevskii, L. A. Slobozhanin, and A. D. Tyuptsov, Low-Gravity Fluid Mechanics (Springer-Verlag, Berlin, 1987).

${ }^{10} \mathrm{G}$. C. Mason, "An experimental determination of the stable length of cylindrical tiquid bubbles," J. Colloid Interface Sci. 32, 172 (1970).

"M. A. Erfe, R. D. Gillette, and D. C. Dyson, "Stability of interfaces of revolution with constant surface tension. The case of the catenoid," Chern. Eng. J. 1, 97 (1970).

${ }^{12}$ R. D. Gitlette and D. C. Dyson, "Stability of fluid interfaces of revolution between equal solid circular plates," Chem. Eng. J. 2, 44 (1971).

${ }^{13}$ I. Da Riva and I. Martínez, "Floating zone stability (Exp. 1-ES-331)," in ESA SP-142 (European Space Agency, Paris, 1979), p. 67.

${ }^{14} \mathrm{~L}$. A. Slobozhanin, "Problems on the stability of liquids in equilibrium, appearing in spatial technology," in Hydromechanics and Heat and Mass Transfer in Zero-Gravity (Nauka, Moscow, 1982), p. 9 (in Russian).

${ }^{15}$ A. Sanz and I. Martínez, "Minimum volume for a liquid bridge between equal disks," J. Colloid Interface Sci. 93, 235 (1983).

${ }^{16} \mathrm{M}$. J. Russo and P. H. Steen, "Instability of rotund capillary bridges to general disturbances, experiment and theory," J. Colloid Interface Sci. 113, 154 (1986).

${ }^{17}$ I. Martínez and J. M. Perales, "Liquid bridge stability data," J. Cryst. Growth 78, 369 (1986).

${ }^{18} \mathrm{~S}$. R. Coriell and M. R. Cordes, "Theory of molten zone shape and stability," J. Cryst. Growth 42, 466 (1977).

${ }^{19} \mathrm{~S}$. R. Coriell, S. C. Hardy, and M. R. Cordes, "Stability of liquid zones," J. Colloid Interface Sci. 60, 126 (1977).

${ }^{20}$ J. M. Vega and J. M. Perales, "Almost cylindrical isorotating liquid bridges for small Bond numbers," in ESA SP-191 (European Space Agency Publication Division, ESTEC, Noordwijk, 1983), p. 247.

${ }^{21}$ L. A. Slobozhanin, "Hydrostatic problems arising during simulation of the process of purification of materials and growth of single crystals by the floating zone method. Part 3: Equilibrium and stability of zone in gravitational field and the field of centrifugal forces," Preprint 25.84, Fiz. Teckh. Inst. Nizk. Temp. Akad. Nauk Ukr. USSR, Kharkov (1984) (in Russian).

${ }^{22}$ L. A. Slobozhanin, "Investigation of hydrostatic problems simulating the zone melting process," Izv. Akad. Nauk USSR Ser. Fiz. 49,652 (1985) (in Russian).

${ }^{23}$ I. V. Barmin, B. E. Vershinin, I. G. Levitina, and A. S. Senchenkov, "Stability of liquid rotating zone," Izv. Akad. Nauk SSSR Ser. Fiz. 49, 661 (1985) (in Russian).

${ }^{24}$ J. Meseguer and A. Sanz, "Numerical and experimental study of the dynamics of axisymmetric slender liquid bridges," J. Fluid Mech. 153, 83 (1985).

${ }^{25}$ I. Martínez, J. M. Haynes, and D. Langbein, "Fluid statics and capillarity," in Fhid Sciences and Material Science in Space (SpringerVerlag, Berlin, 1987), p. 53.

${ }^{26} \mathrm{~J}$. M. Perales, J. Meseguer, and I. Martínez, "Minimum volume stability limits for axisymmetric liquid bridges subject to steady axial acceleration," J. Cryst. Growth 110, 885 (1991).

${ }^{27}$ I. Martínez, "Stability of axisymmetric liquid bridges," in ESA SP-191 (European Space Agency, Paris, 1983), p. 267.

${ }^{28}$ J. Meseguer, "Stability of slender, axisymmetric liquid bridges between unequal disks," J. Cryst. Growth 67, 141 (1984).

${ }^{29} \mathrm{~N}$. A. Bezdenejinykh and J. Meseguer, "Stability limits of minimum volume and breaking of axisymmetric liquid bridges between unequal disks," Microgravity Sci. Technol. 4, 235 (1991).

${ }^{30} \mathrm{~J}$. Meseguer, "The influence of axial microgravity on the breakage of axisymmetric slender liquid bridges," J. Cryst. Growth 62, 577 (1983).

${ }^{31}$ W. Heywang, "Zur Stabilität senkrechter Schmelzzonen," Z. Naturforsch. 11a, 238 (1956).

${ }^{32}$ L. A. Slobozhanin, "Some problems of equilibrium stability of zero- $g$ liquid bridges," in Hydromechanics and Heat/Mass Transfer in Microgravity (Gordon and Breach, Philadelphia, 1992), p. 185.

${ }^{33} \mathrm{~N}$. A. Bezdenejnykh, J. Meseguer, and J. M. Perales, "Experimental analysis of stability limits of capillary liqutid bridges," Phys. Fluids A 4, 677 (1992).

${ }^{34}$ D. Langbein, "Crystal growth from liquid columnns," J. Cryst. Growth 104, 47 (1990). 